\title{
Mitteilungen der DGGEF
}

Gynäkologische Endokrinologie 2019 • 17:298

https://doi.org/10.1007/s10304-019-00284-z

() Springer Medizin Verlag GmbH, ein Teil von Springer Nature 2019

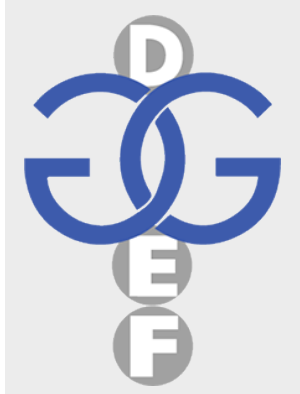

\section{Herausgeber}

Deutsche Gesellschaft für Gynäkologische Endokrinologie

und Fortpflanzungsmedizin e.V.

Prof. Dr. med. Ludwig Kiesel (V.i.S.d.P.)

Klinik für Frauenheilkunde und Geburtshilfe

Universitätsklinikum Münster

Albert-Schweitzer-Campus 1

48149 Münster

Tel: +49 (0) $251-8348202$

Ludwig.Kiesel@ukmuenster.de

www.dggef.de

Redaktion

Vorstand der DGGEF

\section{Verzögerung der}

\section{Menopause durch ovarielle Kryokonservierung?}

\section{Hintergrund}

Ist der Aufschub der Menopause ohne Einnahme von hormoneller Substitutionstherapie nun eine tatsächliche Option? August diesen Jahres präsentierte die britische Firma ProFam (Protecting Fertility and Menopause) ihre neue Methode zur künstlichen Verzögerung der Menopause. ProFam, gegründet von Arri Coomarasamy, Christiani Amorim, Yousri Afifi und Simon Fisher, vier renommierte Reproduktionsmediziner, verspricht mit ihrer Methode die Menopause um bis zu 20 Jahre aufzuschieben und somit Frauen ein Leben frei von Hormonentzugsbeschwerden wie Hitzewallungen, Depressionen und Osteoporose bis hin zum längerem Fertilitätserhalt zu ermöglichen. Hierzu werde jungen gesunden Frauen mittels laparoskopischen Eingriffs ovarielles Gewebe entnommen, bei $-150^{\circ}$ tiefgefroren, um zu Beginn des Klimakteriums wieder eingepflanzt werden zu können. So werde eine fortgesetzte Hormonproduktion garantiert und menopausale Symptome können abgewandt werden. Maximalalter zur Gewebeentnahme sei auf 40 Jahre festgelegt. So beschreibt ProFam das Verfahren, das be- reits an neun gesunden Frauen durchgeführt wurde [1].

Bislang gilt die oben beschriebene Methode der Kryokonservierung als Möglichkeit des Fertilitätserhalts bei Krebspatientinnen, deren Eizellen aufgrund einer aggressiven Chemo- oder Radiotherapie Schaden nehmen könnten und keine Zeit mehr für eine Eizellentnahme bleibt [2]. Betroffene Patientinnen können sich diesbezüglich an das FertiPROTEKT Netzwerk e. V. wenden und finden hier Ansprechpartner und ausgebildete Zentren. [3] Über 130 Kinder sind bereits infolge dieses medizinischen Eingriffs an Krebspatientinnen zur Welt gekommen, 10 davon aus präpubertärem Gewebe. Bereits in vergangenen Jahren wurden Ideen geäußert, diese Art des Fertilitätserhalts auf gesunde Frauen anzuwenden und somit eine natürliche Hormonproduktion auch in höherem Alter zu garantieren. 2014 publizierten Stoop und Kollegen in der Lancet einen Artikel zu eben dieser Idee und stießen hiermit auf vielseitiges, aber kontroverses Interesse der Kollegen [4] Die prinzipielle Idee des operativen Fertilitätserhalts gesunder Frauen sowie der hierdurch gewonnen zeitliche Puffer bis zur Menopause ist daher nicht neu, die Durchführung der Methode erfolgte jedoch erst jetzt.

\section{Kommentar}

Verschiedene Gremien und Gesellschaften haben sich bereits zur präsentierten Methode geäußert. Am 6. August gab die British Menopause Society (BMS) ein Statement heraus, in dem ausdrücklich Bedenken geäußert werden. Gut entwickelte klinisch basierte Studien werden benötigt, bevor die Methode als sicher eingestuft werden könne. Die BMS führt insbesondere an, dass reimplantiertes Gewebe nicht immer zuverlässig funktioniere und die Lebenszeit des Gewebes variabel sei. Eine studienfundierte Nutzenrisikoanalyse sei erforderlich, da es sich um einen operativen Eingriff an gesunden Frauen handele. Ebenso müsse das Risiko für Ovarialkarzinome und Mammakarzinome beurteilt werden. Da bislang noch keine Langzeitanalysen vorliegen, müsse die Einschätzung zur präsentierten Methode vorsichtig erfolgen [5]. Die International Menopause Society (IMS) schließt sich dem Statement der BMS an. Auch das Royal College of Obstetrics and Gynecology (RCOG) positioniert sich skeptisch und hebt hervor, dass die mit einem laparoskopischen Eingriff einhergehenden Risiken wie die Verletzung von Darm, Harnblase oder Harnleiter, nicht vernachlässigt werden sollten. Auch wird betont, dass die heutzutage vorhandene Hormonersatztherapie hoch effektiv, ein- fach und sicher für die meisten Frauen sei und daher die Notwendigkeit eines bis hin zu mehreren operativen Eingriffen zur Abwendung von Menopausebeschwerden hinterfragt werden sollte.

\section{Autor|Kontakt}

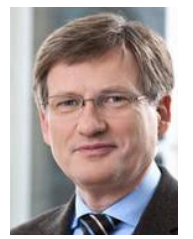

Prof. Dr. med. Ludwig Kiesel Direktor der Klinik für Frauenheilkunde und Geburtshilfe, Universitätsklinikum Münster

Geschäftsstelle DGGEF e.V. Anne Becker, c/o SoftconsuLt Weißdornweg 17, D-35041 Marburg Tel.: +49 (0) 6420 93444, info@dggef.de, www.dggef.de

\section{Literatur}

1. http://www.profam.co.uk. Zugegriffen: 22. Aug. 2019

2. Oktay K et al (2018) Fertility preservation in patients with cancer: ASCO clinical practice guideline update. J Clin Oncol 36(19):1994-2001 (Jul 1)

3. https://fertiprotekt.com. Zugegriffen: 22. Aug. 2019

4. Stoop et al (2014) Fertility preservation for age-related fertility decline. Lancet 384(9950):1311-1319

5. https://thebms.org.uk/2019/08/ new-medical-procedure-coulddelay-menopause-by-twenty-yearsbms-response/. Zugegriffen: 22. Aug. 2019 